\section{OP10 IMPACT OF THE PUBLIC HEALTH RESPONSIBILITY DEAL ON SALT INTAKE, CARDIOVASCULAR DISEASE AND GASTRIC CANCER: INTERRUPTED TIME SERIES AND MICROSIMULATION STUDY}

${ }^{1}$ A Laverty*, ${ }^{2} \mathrm{C}$ Kypridemos, ${ }^{1} \mathrm{P}$ Seferidi, ${ }^{1} \mathrm{EP}$ Vamos, ${ }^{1} \mathrm{f}$ Pearson-Stuttard, ${ }^{2} \mathrm{~B}$ Collins, ${ }^{1} \mathrm{~S}$ Capewell, ${ }^{2} \mathrm{~K}$ Fleming, ${ }^{2} \mathrm{M}$ O'Flaherty, ${ }^{1} \mathrm{C}$ Millett. ${ }^{1}$ Public Health Policy Evaluation Unit, School of Public Health, Imperial College London, London, UK; ${ }^{2}$ Department of Public Health and Policy, University of Liverpool, Liverpool, UK

\subsection{6/jech-2019-SSMabstracts.10}

Background Public-Private Partnerships (PPPs) are increasingly promoted as a key mechanism to improve population health, including addressing dietary risk factors for non-communicable disease. In 2011, England introduced the Public Health Responsibility Deal (RD), a PPP which gave greater freedom to the food industry to set and monitor targets for sodium intakes, and replaced an internationally recognised scheme involving more independent scrutiny. We estimated the impact of the RD on trends in population level sodium intake and associated changes in cardiovascular disease (CVD) and gastric cancer (GCa) incidence and mortality and their economic costs in England from 2011-2025.

Methods We used interrupted time series models with 24-hour urine sample data to assess trends in sodium intake both before and after the implementation of the RD. We used the IMPACT $_{\mathrm{NCD}}$ microsimulation model to estimate impacts of these changes in sodium consumption on CVD and Gastric Cancer incidence, mortality and economic impacts, as well as equity impacts. We modelled a baseline scenario of sodium intake continuing as it had pre-RD and compared observed intakes with this.

Results Between 2003 and2010 mean sodium intake was falling annually by 0.20 grams/day among men and $0.12 \mathrm{~g} / \mathrm{d}$ among women ( $p$ value for trend both <0.001). After RD implementation in 2011, annual declines in sodium intake slowed statistically significantly to $0.11 \mathrm{~g} / \mathrm{d}$ among men and $0.07 \mathrm{~g} / \mathrm{d}$ among women ( $\mathrm{p}$ values for differences in trend both $\mathrm{p}<0.001)$. Weestimated that the RD has been responsible for approximately 9,900 (Interquartile Quartile Range: 6,700 to 13,000) additional cases of CVD and 1,500 (IQR: 510 to 2,300) additional cases of GCa between 2011 and 2018. If the RD continues unchanged between 2019 and 2025, approximately 26,000 (IQR: 20,000 to 31,000) additional cases of CVD and 3,800 (IQR: 2,200 to 5,300) cases of GCa may occur. The economic costs of the RD have been approximately $£ 160$ million (IQR: $£ 88$ to $£ 230$ million) to date, and approximately $£ 970$ million (IQR: $£ 760$ to $£ 1,200$ million) if the RD is continued unchanged until 2025.

Discussion These findings suggest that declines in sodium intake slowed after implementation of the RD in England and this likely generated excess CVD and cancer burdens, plus additional healthcare, and societal costs. Public private partnerships such as the RD which lack robust and independent target setting, monitoring and enforcement are unlikely to produce optimal health gains.

Funding for this research was from the UK Prevention Research Partnership. UKPRP Consortium Development Grant. UKPRP_CO1_105. QUEST: QUantifying Equitable Solutions To prevent Non-Communicable Diseases. The School of Public Health at Imperial College London is grateful for the support of the NIHR SPHR.

\section{Early years and Life course effects}

\section{OP11 PREMATURE MORTALITY IN ADULT SURVIVORS OF CHILD ABUSE AND NEGLECT}

${ }^{1} \mathrm{NT}$ Rogers*, ${ }^{2} \mathrm{C}$ Power, ${ }^{1}$ SP Pereira. ${ }^{1}$ UCL Research Department of Epidemiology and Public Health, London WCIE THB, UK; ${ }^{2}$ Population, Policy and Practice, UCL Great Ormond Street Institute of Child Health, University College London, London, UK

\subsection{6/jech-2019-SSMabstracts.11}

Background Child maltreatment (neglect and abuse) is not uncommon and has established associations with adverse socioeconomic and health outcomes; however there is sparse evidence on its relationship with premature mortality in adulthood. While different types of maltreatment co-occur, previous studies have examined types of maltreatment separately or as a combined score and there is little understanding of possible differential effects. Using a general population birth cohort, we aimed to establish whether different types of maltreatment were associated with risk of all-cause mortality in mid-adulthood.

Methods Using data from the 1958 British cohort $(\mathrm{N}=9311)$, we examined associations between child neglect (prospectively recorded at $7 \mathrm{y}$ and $11 \mathrm{y}$ ) and physical, psychological, witnessing and sexual abuse (retrospectively recorded at $44 / 45 y$ ) with risk of all-cause mortality between 2002/3 (when participants were 44/45y) and December 2016. Death was ascertained through receipt of certificates from the NHS Central Register $(n=296)$ and from information from relatives or close friends $(n=16)$. Associations were examined for each type of maltreatment separately and also combined as a score, to assess cumulative burden, using Cox proportional hazard models with and without adjustment for gender, early-life covariates (e.g. childhood socioeconomic position) and for other maltreatment types to allow for co-occurrence.

Results Approximately 22\% of participants reported at least one type of maltreatment in childhood and $3.4 \%$ had died between 2002/3 and 2016. Individuals identified with neglect $(10.4 \%$ of the population), physical $(6 \%)$ or sexual abuse $(1.6 \%)$ were at increased risk for all-cause mortality during the follow-up period, independent of other types of maltreatments and childhood socioeconomic position: adjusted Hazard Ratios (HRs) were 1.43(95\% CI:1.04,1.95), 1.76(1.11,2.80) and $3.07(1.77,5.33)$ respectively. Childhood psychological and witnessing abuse were not associated with mortality after allowing for other factors. In general, risk of all-cause mortality increased with number of types of maltreatment, with HRs of $1.61(1.21,2.14)$ and $3.43(2.28,5.17)$ for a single and $3+$ types of maltreatments respectively, compared to those with none.

Conclusion Adult survivors of child neglect, physical and sexual abuse are at increased risk of premature mortality in midadulthood. Findings suggest that periodic health assessments for survivors of specific types of child maltreatment over the life-course may help eradicate differences in premature mortality. Child abuse was reported retrospectively in adulthood, although child neglect and potential confounding factors were ascertained prospectively. 